\title{
DOMAINS WITH LIPSCHITZ MAPPING FUNCTIONS
}

\author{
FRANK DAVID LESLEY
}

\section{Introduction}

Suppose that $\Gamma$ is a closed Jordan curve in the $\omega$-plane, with interior $\Omega$ and exterior $\Omega^{*}$. Let $f$ and $f^{*}$ respectively be conformal mappings of $D=\{|\zeta|<1\}$ and $D^{*}=\{|\zeta|>1\}$ onto $\Omega$ and $\Omega^{*}$. Both $f$ and $f^{*}$ may be extended to homeomorphisms between the closures of their domains and images, and we denote these extensions by $f$ and $f^{*}$ also. It is a much studied phenomenon that various geometric properties of $\Gamma$ correspond to properties of the mapping functions or their inverses. In particular, there are many results in which properties of $\Gamma$ imply properties of the mapping functions. There are fewer where one assumes properties of the functions to infer properties of $\Gamma$, and there are still fewer equivalences between behavior of $\Gamma$ and boundary behavior of $f$ or $f^{*}$. In some cases, a condition on $f$ alone (for example) is equivalent to a condition on $\Gamma$. For instance, absolute continuity of $f\left(e^{i \theta}\right)$ is equivalent to the rectifiability of $\Gamma$ (and $f^{*}\left(e^{i \theta}\right)$ is then absolutely continuous as well). Sometimes a "two sided condition", one involving both $f$ and $f^{*}$, is needed. An example of this is Ahlfors' characterization of quasiconformal curves in terms of $f^{*-1} \circ f$ [1, Chapter 4].

In this paper we are interested in consequences of Lipschitz continuity on $\partial D$ of the mapping functions. We first recall a few definitions.

For $\omega_{1}$ and $\omega_{2}$ on $\Gamma$, let $\Gamma\left(\omega_{1}, \omega_{2}\right)$ be the arc of smaller diameter between $\omega_{1}$ and $\omega_{2}$. Then $\Gamma$ is a $c$-quasiconformal curve (or a quasiconformal curve) if there exist positive $c$ and $\delta$ such that for $\left|\omega_{1}-\omega_{2}\right|<\delta$ and $\omega_{0} \in \Gamma\left(\omega_{1}, \omega_{2}\right)$,

$$
\frac{\left|\omega_{1}-\omega_{0}\right|+\left|\omega_{0}-\omega_{2}\right|}{\left|\omega_{1}-\omega_{2}\right|}<c
$$

The curve $\Gamma$ is an asymptotically conformal curve if

$$
\lim _{\left|\omega_{1}-\omega_{2}\right| \rightarrow 0} \sup _{\omega_{0} \in \Gamma\left(\omega_{1}, \omega_{2}\right)} \frac{\left|\omega_{1}-\omega_{0}\right|+\left|\omega_{0}-\omega_{2}\right|}{\left|\omega_{1}-\omega_{2}\right|}=1 .
$$

Suppose now that $\Gamma$ is rectifiable. For $\omega_{1}, \omega_{2} \in \Gamma$; let $\Delta s$ be the length of the shorter arc between $\omega_{1}$ and $\omega_{2}$. Then $\Gamma$ is a bounded arclength-chordlength 
curve, or a quasismooth curve, if there exists $c>0$ such that

$$
\frac{\Delta s}{\left|\omega_{1}-\omega_{2}\right|} \leqq c
$$

for all $\omega_{1}, \omega_{2} \in \Gamma . \quad \Gamma$ is asymptotically smooth if

$$
\lim _{\left|\omega_{1}-\omega_{2}\right| \rightarrow 0} \frac{\Delta s}{\left|\omega_{1}-\omega_{2}\right|}=1
$$

The properties (1.2) and (1.4) have been studied by Pommerenke, who showed them to be equivalent to certain properties of $f([10]$, see also [2], [8]). In [7], Hölder continuity of $f$ and $f^{*}$ (as well as $f^{-1}$ and $f^{*-1}$ ) was inferred from (1.1) with Hölder exponents depending on $c$ (see [9] also). The question then arose as to whether Hölder continuity of all of the mapping functions implied (1.1). This was settled by Becker and Pommerenke [3] who constructed a curve $\Gamma$ for which all four functions are Hölder continuous but which is not a quasiconformal curve.

In this paper we consider the hypothesis that $f$ and $f^{*}$ are Lipschitz continuous and ask what can be said about $\Gamma$.

We begin by remarking that this implies Lipschitz continuity of $f^{-1}$ and $f^{*-1}$ on $\Gamma$ [6]. From the Lipschitz continuity of $f$ and $f^{-1}$ it is easy to see that (1.3) holds; $\Gamma$ is quasismooth. Our main result is Theorem 3. If $\Gamma$ is a quasiconformal curve and $f$ and $f^{*}$ are locally Lipschitz at $\zeta_{0} \in \partial D$ with $f\left(\zeta_{0}\right)=f^{*}\left(\zeta_{0}\right)=\omega_{0}$, then a local version of (1.2) holds. This "local asymptotic conformality" has been studied by Rodin and Warschawski [12] and has several consequences, both for the mapping functions and for $\Gamma$. For example, the "Visser-Ostrowski limit" for $f$ exists and equals 1 at $\zeta_{0}$ (Corollary 1). As another example, near $\omega_{0}, \Gamma$ may be sandwiched between two asymptotically smooth arcs containing $\omega_{0}$ (Corollary 2).

If $f$ and $f^{*}$ are uniformly Lipschitz continuous on $\partial D$ then $\Gamma$ is locally asymptotically conformal at every point of $\Gamma$, so that one might expect (1.2) to hold, since the hypothesis is a uniform one. However, in Section 3 we construct a curve $\Gamma$ for which $f$ and $f^{*}$ are Lipschitz but for which (1.2) fails. In fact, for any $\alpha>1, \Gamma$ may be constructed so that the limit in (1.2) is at least $\alpha$. In other words, the $c$ in (1.1) cannot be taken to be smaller than $\alpha$, no matter how small $\delta$ is.

In both sections we work with mappings between strip domains which correspond to $f$ and $f^{*}$, and the principal tools are the Ahlfors inequalities. In Section 2 we show that the Lipschitz continuity of $f$ and $f^{*}$ at $\xi_{0}$ implies that the strips corresponding to $\Omega$ and $\Omega^{*}$ have width nearly $\pi$ in a certain sense (Theorem 1). Then we show that the strips both have the "Ahlfors distortion property" which means that the real parts of the strip mappings are given asymptotically by the "Ahlfors integral" (Theorem 2). The geometric consequences of this property [11], together with our Theorem 1 then yield our main result. 
None of the three results follows from the hypothesis that $f, f^{-1}$ and $f^{*-1}$ are all Lipschitz continuous, as is seen from Theorem 2 in [6].

I would like to thank Burt Rodin, Uri Srebro and Steve Warschawski for several enlightening discussions.

\section{Consequences of Lipschitz continuity}

In all that follows we assume that $f$ and $f^{*}$ are conformal mappings of $D=\{|\zeta|<1\}$ and $D^{*}=\{|\zeta|>1\}$ onto $\Omega=\operatorname{Int} \Gamma$ and $\Omega^{*}=$ Ext $\Gamma$ respectively. $\Gamma$ is a closed Jordan curve and the functions $f$ and $f^{*}$ are extended continuously to the boundaries of their domains. Without loss of generality in what follows, we assume that for a certain $\zeta_{0} \in \partial D, f\left(\zeta_{0}\right)=\omega_{0}=f^{*}\left(\zeta_{0}\right), f\left(i \zeta_{0}\right)=\omega_{0}^{\prime}=f^{*}\left(i \zeta_{0}\right)$ and $f\left(-\zeta_{0}\right)=\omega_{0}^{\prime \prime}=f^{*}\left(-\zeta_{0}\right)$. We then transform $\Omega$ and $\Omega^{*}$ into strip domains via

$$
w(\omega)=\log \frac{\left(\omega_{0}^{\prime \prime}-\omega\right)}{\left(\omega_{0}-\omega\right)}
$$

where the branch of the logarithm in both $\Omega$ and $\Omega^{*}$ is chosen so that for $w_{0}^{\prime}=w\left(\omega_{0}^{\prime}\right)$, we have $\operatorname{Im} w_{0}^{\prime} \in(-\pi, \pi]$.

The domain $\Omega$ corresponds to a strip $\Sigma_{1}$ which is bounded by two Jordan arcs $C_{1}$ and $C_{2}$, each with $-\infty$ and $+\infty$ as endpoints. Let $C_{1}$ be the arc containing $w_{0}^{\prime}$. Then $\Omega^{*}$ corresponds to a strip $\Sigma_{2}$ bounded by $C_{1}$ and $C_{2}^{\prime}=$ $\left\{w+2 \pi i, w \in C_{2}\right\}$. Letting $z=\log \left(\left(\zeta_{0}+\zeta\right) /\left(\zeta_{0}-\zeta\right)\right)$ for suitable branches of the logarithm, $D$ corresponds to $S_{1}=\{z=x+i y ;|y|<\pi / 2\}$ and $D^{*}$ corresponds to $S_{2}=\{z=x+i y: \pi / 2<y<3 \pi / 2\}$. The mappings $f$ and $f^{*}$ correspond to mappings $w_{1}(z)$ and $w_{2}(z)$ between $S_{1}$ and $\Sigma_{1}$, and $S_{2}$ and $\Sigma_{2}$ respectively. We denote their inverses by $z_{1}(w)$ and $z_{2}(w)$. The mappings extend continuously to the closures of their domains and we use the same names for the extensions. Note that $w_{1}(-\infty)=$ $w_{2}(-\infty)=-\infty, w_{1}(+\infty)=w_{2}(+\infty)=+\infty$, and $w_{1}(\pi i / 2)=w_{2}(\pi i / 2)=w_{0}^{\prime}$.

Next, we let $\Lambda_{u}$ be the vertical line $\{w: \operatorname{Re} w=u\}$. For each $i=1,2, \Sigma_{i}-\Lambda_{u}$ contains a component $\Sigma_{i}^{\prime}$ in $\{\operatorname{Re} w<u\}$ which has $-\infty$ as a boundary point. Let $\sigma_{i}(u)$ be the maximal subinterval of $\Lambda_{u} \cap \bar{\Sigma}_{i}$ which is a common boundary arc in $\bar{\Sigma}_{i}$ of $\bar{\Sigma}_{i}^{\prime}$ and the component of $\bar{\Sigma}_{i}-\bar{\Sigma}_{i}^{\prime}$ with $+\infty$ as a boundary point. We let $\theta_{i}(u)$ be the length of $\sigma_{i}(u)$ and we note that $\theta_{1}(u)+\theta_{2}(u) \leqq 2 \pi$.

We recall the Ahlfors distortion theorem [5]: fix $i$ and define

$$
\underline{x}_{i}(u)=\min _{w \in \sigma_{i}(u)} x_{i}(w) \quad \text { and } \quad \bar{x}_{i}(u)=\max _{w \in \sigma_{i}(u)} x_{i}(w),
$$

where $x_{i}(w)=\operatorname{Re} z_{i}(w)$. For $u^{\prime}<u$, if

$$
\int_{u^{\prime}}^{u} \frac{d t}{\theta_{i}(t)}>1
$$


then

$$
\underline{x}_{i}(u)-\bar{x}_{i}\left(u^{\prime}\right) \geqq \pi \int_{u^{\prime}}^{u} \frac{d t}{\theta_{i}(t)}-2 \pi .
$$

The following lemma characterizes the Lipschitz continuity of $f, f^{-1}, f^{*}$ and $f^{*-1}$ in terms of the strip mappings (see [6]). We state the result for $f$; a similar statement holds for $f^{*}$. In all that follows $M, M_{1}, M_{2}, \ldots, K, K_{1}, K_{2}, \ldots$ denote constants. The same symbols may be used for different constants in the statements of different results.

Lemma 1. Suppose that $\left|f(\zeta)-f\left(\zeta_{0}\right)\right| \leqq K_{1}\left|\zeta-\zeta_{0}\right|$ for $\zeta_{0} \in \partial D$ and all $\zeta \in \bar{D}$. Then there exist $M_{1}$ and $M_{2}$ such that for $w=u+i v \in \bar{\Sigma}_{1}$ with $u>0$, we have

$$
\begin{aligned}
& x_{1}(w)-u \leqq M_{1} \text { and } \\
& \int_{0}^{u} \frac{\pi-\theta_{1}(t)}{\theta_{1}(t)} d t \leqq M_{2} .
\end{aligned}
$$

If $\left|f^{-1}(\omega)-f^{-1}\left(\omega_{0}\right)\right| \leqq K_{2}\left|\omega-\omega_{0}\right|$ for $\omega_{0} \in \Gamma$ and all $\omega \in \bar{\Omega}$ then there exists $M_{3}$ for which

$$
M_{3} \leqq x_{1}(w)-u .
$$

The lemma is stated locally, but if $f$ and $f^{-1}$ are uniformly Lipschitz continuous in $\bar{D}$ and $\bar{\Omega}$ respectively, then $M_{1}, M_{2}$ and $M_{3}$ are independent of $\zeta_{0}$.

We need a sharpening of the inequality between the harmonic and arithmetic means.

Lemma 2. Suppose that $\theta_{1}, \theta_{2}, \ldots, \theta_{n}$ are positive real numbers for which $\sum_{i=1}^{n} \theta_{i} \leqq 2 \pi$, and that $\bar{\theta}=(1 / n) \sum_{i=1}^{n} \theta_{i}$. Then

$$
\sum_{i=1}^{n} \frac{1}{\theta_{i}} \geqq \frac{1}{\bar{\theta}}\left(n+\frac{1}{\pi^{2}}\left(\sum_{i=1}^{n}\left(\theta_{i}-\frac{2 \pi}{n}\right)^{2}\right)-\frac{n}{\pi^{2}}\left(\bar{\theta}-\frac{2 \pi}{n}\right)^{2}\right) .
$$

Proof. We recall a well known identity of Lagrange. For $a_{1}, \ldots, a_{n}$ and $b_{1}, \ldots, b_{n}$ real,

$$
\left(\sum_{i=1}^{n} a_{i} b_{i}\right)^{2}=\left(\sum_{i=1}^{n} a_{i}^{2}\right)\left(\sum_{i=1}^{n} b_{i}^{2}\right)-\frac{1}{2} \sum_{j, k=1}^{n}\left(a_{j} b_{k}-a_{k} b_{j}\right)^{2} .
$$

Letting $a_{i}=\sqrt{\theta_{i}}$ and $b_{i}=1 / \sqrt{\theta_{i}}$, and noting that $\theta_{j} \theta_{k} \leqq \pi^{2}$, we have

$$
\begin{aligned}
\sum_{i=1}^{n} \frac{1}{\theta_{i}} & =\frac{1}{\sum_{i=1}^{n} \theta_{i}}\left(n^{2}+\frac{1}{2} \sum_{j, k=1}^{n} \frac{\left(\theta_{j}-\theta_{k}\right)^{2}}{\theta_{j} \theta_{k}}\right) \\
& \geqq \frac{1}{\bar{\theta}}\left(n+\frac{1}{2 n} \sum_{j, k=1}^{n} \frac{\left(\left(\theta_{j}-2 \pi / n\right)-\left(\theta_{k}-2 \pi / n\right)\right)^{2}}{\pi^{2}}\right) \\
& =\frac{1}{\bar{\theta}}\left(n+\frac{1}{\pi^{2}} \sum_{i=1}^{n}\left(\theta_{i}-\frac{2 \pi}{n}\right)^{2}-\frac{1}{n \pi^{2}} \sum_{j, k=1}^{n}\left(\theta_{j}-\frac{2 \pi}{n}\right)\left(\theta_{k}-\frac{2 \pi}{n}\right)\right) .
\end{aligned}
$$

The lemma follows by summing the last term. 
Theorem 1. Let $\Gamma$ be a closed Jordan curve and let $f$ and $f^{*}$ be conformal mappings of $D$ and $D^{*}$ onto $\Omega=\operatorname{Int} \Gamma$ and $\Omega^{*}=\operatorname{Ext} \Gamma$ respectively. Suppose that $f$ and $f^{*}$ are Lipschitz continuous at $\zeta_{0} \in \partial D$, with $f\left(\zeta_{0}\right)=f^{*}\left(\zeta_{0}\right)=\omega_{0}$. Then there exists $M$ such that for $\theta_{1}(u)$ and $\theta_{2}(u)$ as above,

$$
\sum_{i=1}^{2} \int_{0}^{\infty}\left(\theta_{i}(u)-\pi\right)^{2} d u<M
$$

Proof. By (2.3) applied to $f$ and $f^{*}$, there exists $M_{1}$ such that for $\bar{\theta}(t)=$ $\left(\theta_{1}(t)+\theta_{2}(t)\right) / 2$ and for all $u>0$,

$$
\begin{gathered}
M_{1} \geqq \int_{0}^{u} \frac{\pi}{\theta_{1}(t)}+\frac{\pi}{\theta_{2}(t)}-\frac{2 \bar{\theta}(t)}{\bar{\theta}(t)} d t \\
\geqq \int_{0}^{u} \frac{2(\pi-\bar{\theta}(t))}{\bar{\theta}(\bar{t})} d t+\frac{1}{\pi} \int_{0}^{u} \frac{1}{\bar{\theta}(t)}\left(\sum_{i=1}^{2}\left(\theta_{i}(t)-\pi\right)^{2}\right) d t-\frac{2}{\pi} \int_{0}^{u} \frac{(\bar{\theta}(t)-\pi)^{2}}{\bar{\theta}(t)} d t,
\end{gathered}
$$

by Lemma 2. From the proof of Lemma 2 we see that the difference of the last two integrals is non-negative so that

from which it follows that

$$
\int_{0}^{\infty} \frac{(\pi-\bar{\theta}(t))}{\bar{\theta}(t)} d t \leqq \frac{M_{1}}{2},
$$

$$
\int_{0}^{\infty} \frac{(\pi-\bar{\theta}(t))^{2}}{\bar{\theta}(t)} d t \leqq \frac{M_{1} \pi}{2} .
$$

Applying this to (2.7) we obtain (2.6), with $M=2 M_{1} \pi^{2}$.

Theorem 2. Under the conditions of Theorem 1, each $\Sigma_{i}$ has the Ahlfors distortion property: For $w=u+i v$ and $w^{\prime}=u^{\prime}+i v^{\prime} \in \Sigma_{i}$

$$
\left|x_{i}(w)-x_{i}\left(w^{\prime}\right)-\int_{u^{\prime}}^{u} \frac{\pi d t}{\theta_{i}(t)}\right|=o(1)
$$

where $o(1) \rightarrow 0$ as $u>u^{\prime} \rightarrow \infty$, uniformly in $v$ and $v^{\prime}$.

Proof. We work with $\Sigma_{1}$, the proof for $\Sigma_{2}$ being identical. It suffices to show that for some $M$,

$$
x_{1}(w)-\int_{0}^{u} \frac{\pi}{\theta_{1}(t)} d t \leqq M,
$$

since by a result of Eke [4, Theorem 2], this implies that for some $\beta$,

$$
x_{1}(w)--\int_{0}^{u} \frac{\pi}{\theta_{1}(t)} d t \rightarrow \beta \quad \text { as } \quad u \rightarrow \infty,
$$

from which (2.8) follows. 
Now, by (2.2) applied to $x_{1}$ and $x_{2}$, and by the distortion theorem, we have

$$
x_{1}(w)-u \leqq u-x_{2}(w)+M_{3} \leqq \int_{0}^{u} \frac{\theta_{2}(t)-\pi}{\theta_{2}(t)} d t+M .
$$

From the inequality $\left(\theta_{2}-\pi\right) / \theta_{2} \leqq\left(\pi-\theta_{1}\right) / \theta_{1}$, it follows that

so that

$$
x_{1}(w)-u \leqq \int_{0}^{u} \frac{\pi-\theta_{1}(t)}{\theta_{1}(t)} d t+M_{4}
$$

$$
x_{1}(w) \leqq \int_{0}^{u} \frac{\pi}{\theta_{1}(t)} d t+M_{4},
$$

and Theorem 2 is proved.

The inequality (2.8) has many geometric consequences, which are studied extensively in [11]. The first result which we will use is the following. Fix $i$ and let $H_{t}=\left\{w \in \Sigma_{i}: y_{i}(w)=t\right\}$. Here $|t|<\pi / 2$ for $\Sigma_{1}$ and $\pi / 2<t<3 \pi / 2$ for $\Sigma_{2}$. Then for $u_{0}$ sufficiently large the level line $H_{t} \cap\left\{w=u+i v: u>u_{0}\right\}$ is the graph of a function $v=\varphi_{t}(u)$ for which $\varphi_{t}^{\prime}(u) \rightarrow 0$ as $u \rightarrow \infty$, [11, Theorem 7]. We will use this result and Theorem 1 to show that $\Gamma$ is locally asymptotically conformal at $\zeta_{0}$, assuming that $\Gamma$ is a quasiconformal curve. To this end we need to know that the functions $w_{i}(z)$ are uniformly continuous for large $x$. This will follow from the next two lemmas.

Lemma 3. Suppose that $\Gamma$ is a bounded quasiconformal curve. Let $\Sigma$ be the image of $\Omega=\operatorname{Int} \Gamma$ under

$$
w=\log \left(\left(\omega-\omega_{0}^{\prime \prime}\right) /\left(\omega-\omega_{0}\right)\right),
$$

for $\omega_{0}$ and $\omega_{0}^{\prime \prime}$ on $\Gamma$ as above and suppose that $\Sigma$ is bounded by the curves $C_{1}$ and $C_{2}$, each with $-\infty$ and $+\infty$ as endpoints. Then there exist $\delta_{0}>0$ and $K>1$ such that for $w_{1}, w_{2}, w_{3} \in C_{1}$ (or all on $C_{2}$ ) with $w_{2}$ between $w_{1}$ and $w_{3}$ and $\left|w_{1}-w_{3}\right|<\delta_{0}$, we have

$$
\frac{\left|w_{1}-w_{2}\right|}{\left|w_{1}-w_{3}\right|}<K
$$

Proof. Let $\zeta=\left(\omega-\omega_{0}\right) /\left(\omega-\omega_{0}^{\prime \prime}\right)$ and let $C$ be the image of $\Gamma$ under this mapping. Then $C$ is also a quasiconformal curve so that there exists $x>1$ such that for $\zeta_{1}, \zeta_{2}, \zeta_{3} \in C$ with $\zeta_{2}$ on the finite $\operatorname{arc} \overparen{\zeta_{1} \zeta_{3}}$, we have ([1], Chapter 4),

$$
\left|\zeta_{1}-\zeta_{2}\right| /\left|\zeta_{1}-\zeta_{3}\right|<x \text {. }
$$

Now let $w_{1}, w_{2}, w_{3}$ be on $C_{1}$ with $w_{j}=\log \left(1 / \zeta_{j}\right)$. Then

$$
\frac{\left|w_{1}-w_{2}\right|}{\left|w_{1}-w_{3}\right|}=\frac{\left|\log \left(\zeta_{2} / \zeta_{1}\right)\right|}{\left|\log \left(\zeta_{3} / \zeta_{1}\right)\right|} \leqq \frac{3\left|\zeta_{2} / \zeta_{1}-1\right|}{\left|\zeta_{3} / \zeta_{1}-1\right|} \leqq \frac{3\left|\zeta_{2}-\zeta_{1}\right|}{\left|\zeta_{3}-\zeta_{1}\right|}<3 \varkappa
$$


as long as

$$
\left|\frac{\zeta_{2}}{\zeta_{1}}-1\right|<\frac{1}{3} \text { and } \quad\left|\frac{\zeta_{3}}{\zeta_{1}}-1\right|<\frac{1}{3} .
$$

To guarantee (2.10) we note that by (2.9) it suffices to have

$$
\left|\frac{\zeta_{3}}{\zeta_{1}}-1\right|<\frac{1}{3 \varkappa} \text {. }
$$

On account of the inequality

$$
\left|\frac{\zeta_{3}}{\zeta_{1}}-1\right|=\left|e^{w_{1}-w_{3}}-1\right| \leqq \frac{\left|w_{1}-w_{3}\right|}{1-\left|w_{1}-w_{3}\right|} \leqq 2\left|w_{1}-w_{3}\right|
$$

for $\left|w_{1}-w_{3}\right|<1 / 2$, it follows that (2.11) is true for

$$
\left|w_{1}-w_{3}\right| \leqq \frac{1}{6 x}=\delta_{0}
$$

Lemma 4. Let $\Gamma$ be a bounded quasiconformal curve and let $f$ map $D$ conformally onto $\Omega=\operatorname{Int} \Gamma$. For $\zeta_{0} \in \partial D$ and $\omega_{0}=f\left(\zeta_{0}\right) \in \Gamma$, suppose that $f$ and $f^{-1}$ are Lipschitz continuous at $\zeta_{0}$ and $\omega_{0}$ respectively. Let $S$ and $\Sigma$ be the corresponding strip domains with $w(z)$ mapping $S$ onto $\Sigma$ as above. Then there exists a constant $M$ such that for any $\varepsilon>0$, there exists $\delta>0$ such that for $z_{0}=x_{0}+i y_{0} \in \partial S$ with $x_{0} \geqq M$ and $z^{\prime \prime} \in \bar{S}$ with $\left|z^{\prime \prime}-z_{0}\right| \leqq \delta$, we have $\left|w\left(z_{0}\right)-w\left(z^{\prime \prime}\right)\right|<\varepsilon$.

Proof. We begin by citing a slight modification of Lemma 1 in [13]: For $z_{0} \in \partial S$ and $r, 0<r<1$, let $k_{r}$ be the semicircle $\left\{\left|z-z_{0}\right|=r\right\} \cap S$. Then for every such $r<1$ there exists a $\varrho_{1}$ with $r \leqq \varrho_{1} \leqq r^{1 / 2}$ such that the image of $k_{\varrho_{1}}$ is a crosscut of $\Sigma$ with length

$$
l_{\varrho_{1}} \leqq\left(\frac{2 \pi A_{z_{0}, r}}{\log (1 / r)}\right)^{1 / 2}
$$

where $A_{z_{0}, \boldsymbol{r}}$ is the area of $T_{\boldsymbol{r}}=w\left(\left\{\left|z-z_{0}\right|<r, z \in S\right\}\right)$.

We must now show that $A_{z_{0}, r}$ is uniformly bounded for all sufficiently large $x_{0}$.

As in our Lemma 1, the local Lipschitz continuity of $f$ and $f^{-1}$ implies the existence of constants $M, M_{1}$, and $M_{3}$ such that for $x>M-1$ and $z=x+i y \in \bar{S}$, we have

$$
M_{3} \leqq x-u(z) \leqq M_{1} .
$$

Thus for $x_{0}>M$ and $x_{0}+r \geqq x \geqq x_{0}-r$ we have

$$
x_{0}+r-M_{3} \geqq u(z) \geqq x_{0}-r-M_{1}
$$

and $T_{r}$ is contained in a vertical strip of width $2 r+\left(M_{1}-M_{3}\right)$. Since the length of $\Sigma \cap l$ does not exceed $2 \pi$ for any vertical line $l$, it follows that for $x_{0}>M$,

$$
A_{z_{0}, r} \leqq 2 \pi\left(2 r+M_{1}-M_{3}\right)
$$


so that

$$
l_{\varrho_{1}} \leqq\left(\frac{4 \pi^{2}\left(2 r+M_{1}-M_{3}\right)}{\log (1 / r)}\right)^{1 / 2} .
$$

Now, let $T_{\varrho_{1}}=w\left(\left\{\left|z-z_{0}\right|<\varrho_{1}, z \in S\right\}\right)$ so that $T_{r} \subset T_{\varrho_{1}}$. Let $w_{1}$ and $w_{3}$ be the endpoints of the image of $k \varrho_{1}$ on $C_{1}$ (or $C_{2}$ ) and let $C^{\prime}$ be the arc of $C_{1}$ (or $C_{2}$ ) with $w_{1}$ and $w_{3}$ as endpoints. Then, for $\left|z_{0}-z^{\prime \prime}\right|=r$ we have

$$
\left|w\left(z_{0}\right)-w\left(z^{\prime \prime}\right)\right| \leqq \operatorname{diam} T_{\boldsymbol{r}} \leqq \operatorname{diam} T_{\varrho_{\mathbf{1}}} \leqq \operatorname{diam} C^{\prime}+l_{\varrho_{1}} .
$$

For $\delta_{0}$ and $K$ as in Lemma 3, choose $\delta>0$ so small that $r<\delta$ implies that $l_{\varrho_{1}}<\max \left(\delta_{0}, \varepsilon /(2 K+1)\right)$. Since $\left|w_{1}-w_{3}\right|<l_{\varrho_{1}}$ we apply Lemma 3 to see that

$$
\left|w\left(z_{0}\right)-w\left(z^{\prime \prime}\right)\right| \leqq 2 K l_{\varrho_{1}}+l_{\varrho_{1}}<\varepsilon,
$$

which proves Lemma 4. We may now prove

Theorem 3. Assume the conditions of Theorem 1 and that $\Gamma$ is a quasiconformal curve. Then $\Gamma$ is asymptotically conformal at $\omega_{0}:$ As $\omega_{1}$ and $\omega_{2}$ approach $\omega_{0}$ from opposite sides

$$
\lim _{\omega_{i} \rightarrow \omega_{0}} \frac{\left|\omega_{1}-\omega_{0}\right|+\left|\omega_{0}-\omega_{2}\right|}{\left|\omega_{1}-\omega_{2}\right|}=1 .
$$

Proof. We may assume that $\omega_{0}=0$. We work with $w_{1}(z): S_{1} \rightarrow \Sigma_{1}$, which we shall refer to as $w(z)$, with inverse $z(w)$. Then for $w\left(\omega_{j}\right)=w_{i}=u_{j}+i v_{j} \in C_{j}$, $j=1,2$, we have

$$
\frac{\left|\omega_{1}\right|+\left|\omega_{2}\right|}{\left|\omega_{1}-\omega_{2}\right|}=\frac{1+\left|\frac{1-e^{w_{1}}}{1-e^{w_{2}}}\right|}{\left|1-\frac{1-e^{w_{1}}}{1-e^{w_{2}}}\right|}=\frac{1+e^{u_{1}-u_{2}}}{\left|1+e^{u_{1}-u_{2}+i\left(v_{1}-v_{2}-\pi\right)}\right|}+o(1)
$$

where $o(1) \rightarrow 0$ as $u_{1}, u_{2} \rightarrow \infty$. We may assume that $u_{1} \geqq u_{2}$ so that it suffices to show that

$$
\lim _{u_{2} \rightarrow \infty} \frac{1+e^{u_{1}-u_{2}}}{\left|1+e^{u_{1}-u_{2}+i\left(v_{1}-v_{2}-\pi\right)}\right|}=1 .
$$

To this end, given $\varepsilon>0$ we first choose $a \geqq 0$ such that $u_{1}-u_{2} \geqq a$ implies that

$$
\frac{1+e^{u_{1}-u_{2}}}{\left|1+e^{u_{1}-u_{2}+i\left(v_{1}-v_{2}-\pi\right)}\right|}<1+\varepsilon,
$$

for any $v_{1}, v_{2}$.

Next, choose $b$ such that $0 \leqq u_{1}-u_{2} \leqq a$ and $\left|v_{1}-v_{2}-\pi\right| \leqq b$ yields (2.12). Finally we show that there exists a real $c$ such that $u_{2} \geqq c$ and $u_{1}-u_{2} \leqq a$ implies that $\left|v_{1}-v_{2}-\pi\right| \leqq b$. By Lemma 4 there exist $M$ and $\delta>0$, such that for $z=x+i y \in \partial S_{1}$ with $x \geqq M$, and for $z^{\prime \prime} \in \bar{S}_{1}$ with $\left|z-z^{\prime \prime}\right| \leqq \delta$, we have

$$
\left|w(z)-w\left(z^{\prime \prime}\right)\right|<\frac{b}{14}
$$


Next choose $u_{0}>M$ so large that for $u \geqq u_{0}$, the level curves $H_{\pi / 2-\delta}$ and $H_{-\pi / 2+\delta}$ are given by the graphs of functions $\varphi_{\pi / 2-\delta}(u)$ and $\varphi_{-\pi / 2+\delta}(u)$, with $\left|\varphi_{\pi / 2-\delta}^{\prime}(u)\right|$ and $\left|\varphi_{-\pi / 2+\delta}^{\prime}(u)\right|$ both less than or equal to $K_{1}=\max (b /(7 a), 1)$.

Suppose that $w=u+i v \in C_{1}$ and $w^{\prime}=u+i v^{\prime} \in H_{\pi / 2-\delta}$. Then

$$
\left|v-v^{\prime}\right| \leqq \frac{b}{7}
$$

The inequality (2.13) also holds for $w=u+i v \in C_{2}$ and $w^{\prime}=u+i v^{\prime} \in H_{-\pi / 2+\delta}$.

To establish (2.13), suppose that $z(w)=x+i \pi / 2=z, z\left(w^{\prime}\right)=x^{\prime}+i(\pi / 2-\delta)=z^{\prime}$, $z^{\prime \prime}=x+i(\pi / 2-\delta)$ and $w^{\prime \prime}=w\left(z^{\prime \prime}\right)$. Then

$$
\left|v-v^{\prime}\right| \leqq\left|v-v^{\prime \prime}\right|+\left|v^{\prime \prime}-v^{\prime}\right| \leqq\left|w-w^{\prime \prime}\right|+K_{1}\left|u^{\prime \prime}-u\right| \leqq 2\left|w-w^{\prime \prime}\right| \leqq \frac{b}{7} .
$$

Now, by Theorem 1 we may choose $U_{0}$ such that $u \geqq U_{0}$ implies that

$$
\int_{U_{0}}^{\infty}\left(\theta_{1}(t)-\pi\right)^{2} d t \leqq\left(\frac{b}{7}\right)^{2} a .
$$

We then let $c=\max \left(u_{0}, U_{0}\right)$ and suppose that $u_{1} \geqq u_{2} \geqq c, \quad w_{1}=u_{1}+i v_{1} \in C_{1}$, $w_{2}=u_{2}+i v_{2} \in C_{2}$ and $u_{1}-u_{2} \leqq a$. We then choose $u_{1}+i v_{1}^{\prime} \in H_{\pi / 2-\delta}$ and $u_{2}+i v_{2}^{\prime} \in$ $H_{-\pi / 2+\delta}$. By (2.14) there exists $u_{3} \in\left(u_{2}, u_{2}+a\right)$ such that $\left|\theta_{1}\left(u_{3}\right)-\pi\right| \leqq b / 7$. Taking $u_{3}+i v_{3} \in C_{1}$ and $u_{3}+i v_{4} \in C_{2}$ to be endpoints of $\sigma\left(u_{3}\right)$, with $u_{3}+i v_{3}^{\prime} \in H_{\pi / 2-\delta}$ and $u_{3}+i v_{4}^{\prime} \in H_{-\pi / 2+\delta}$, we finally have

$$
\begin{aligned}
& \left|v_{1}-\left(v_{2}+\pi\right)\right| \leqq\left|v_{1}-v_{1}^{\prime}\right|+\left|v_{1}^{\prime}-v_{3}^{\prime}\right|+\left|v_{3}^{\prime}-v_{3}\right| \\
& +\left|v_{3}-\left(v_{4}+\pi\right)\right|+\left|v_{4}-v_{4}^{\prime}\right|+\left|v_{4}-v_{2}^{\prime}\right|+\left|v_{2}^{\prime}-v_{2}\right| \\
& \leqq \frac{b}{7}+K_{1} a+\frac{b}{7}+\frac{b}{7}+\frac{b}{7}+K_{1} a+\frac{b}{7} \leqq b,
\end{aligned}
$$

which proves Theorem 3 .

The following are consequences of Theorem 3 which follow from results in [12]. The first is an immediate application of our result and Theorem 7 of [12].

Corollary 1. Assume the hypotheses of Theorem 3. Then

$$
\lim _{\substack{\zeta \rightarrow \zeta_{0} \\ \zeta \in D}} \frac{f^{\prime}(\zeta)\left(\zeta-\zeta_{0}\right)}{f(\zeta)-f\left(\zeta_{0}\right)}=1
$$

for nontangential approach. The corresponding result holds for $f^{*}$.

Corollary 2. Assume the hypotheses of Theorem 3. Then $\Omega \cup\left\{\omega_{0}\right\}$ and $\Omega^{*} \cup\left\{\omega_{0}\right\}$ each contain an open asymptotically smooth arc containing $\omega_{0}$.

As we shall see, $\Gamma$ need not itself be asymptotically smooth. The foregoing means, however, that near $\omega_{0}, \Gamma$ is trapped between asymptotically smooth arcs. 
Proof. Recall the definition of an $L$-strip. $R$ is called an $L$-strip of inclination 0 if

$$
R=\left\{w=u+i v: a<u<\infty, \varphi_{-}(u)<v<\varphi_{+}(u)\right\}
$$

where $a \geqq-\infty$ and $\varphi_{+}, \varphi_{-}$are continuous functions such that for $u^{\prime}>u$,

$$
\lim _{u \rightarrow+\infty} \frac{\varphi_{ \pm}\left(u^{\prime}\right)-\varphi_{ \pm}(u)}{u^{\prime}-u}=0 .
$$

By a construction of Rodin and Warschawski [12, p. 135], the conclusion of Theorem 3 implies that each of $\Sigma_{1}$ and $\Sigma_{2}$ contains an $L$-strip of inclination 0 . In fact, if $R_{1}$ is the $L$-strip contained in $\Sigma_{1}$ with boundary curves given by the graphs of $\varphi_{1+}$ and $\varphi_{1_{-}}$, each of $\varphi_{1+}$ and $\varphi_{1-}$ may be taken to be smooth, with

and

$$
\varphi_{1+}^{\prime}(u) \rightarrow 0 \text { and } \varphi_{1-}^{\prime}(u) \rightarrow 0
$$

$$
\varphi_{1+}(u)-\varphi_{1-}(u) \rightarrow \pi \text { as } u \rightarrow \infty .
$$

The image of the graphs of $\varphi_{1+}$ and $\varphi_{1-}$ in the $\omega$ plane is an asymptotically smooth arc in $\Omega \cup\left\{\omega_{0}\right\}$, containing $\omega_{0}$. Similarly, the boundary of the $L$-strip contained in $\Sigma_{2}$ corresponds to the asymptotically smooth arc in $\Omega^{*} \cup\left\{\omega_{0}\right\}$ which contains $\omega_{0}$.

If the functions $f$ and $f^{*}$ are uniformly Lipschitz continuous on $\Gamma$, then the curve $\Gamma$ is locally asymptotically conformal at every point. One might then ask whether $\Gamma$ is then asymptotically conformal, or in light of Corollary 2, even asymptotically smooth. In the next section we shall see that $\Gamma$ may fail to be asymptotically conformal.

\section{Construction of an example}

We again use mappings between strip domains, and we shall use the same notations as in Section 2. We shall construct a strip domain $\Sigma_{1}$ in the $w=u+i v$ plane, bounded by Jordan arcs $C_{1}$ and $C_{2}$, each with $-\infty$ and $+\infty$ as endpoints, i.e. $C_{j}$ may be given parametrically by $w=w_{j}(t),-\infty<t<+\infty$, with $\operatorname{Re}_{w_{j}}(t) \rightarrow-\infty$ as $t \rightarrow-\infty$ and $\operatorname{Re} w_{j}(t) \rightarrow+\infty$ as $t \rightarrow+\infty$. As above $\Sigma_{2}$ will be the "complementary" domain bounded by $C_{1}$ and $C_{2}^{\prime}=\left\{w+2 \pi i: w \in C_{2}\right\}$. We consider the conformal mappings $w_{1}(z)$ and $w_{2}(z)$ from $S_{1}$ and $S_{2}$ onto $\Sigma_{1}$ and $\Sigma_{2}$ respectively with $w_{j}(-\infty)=-\infty, w_{j}(+\infty)=+\infty$, and $w_{j}(\pi i / 2)=\pi i / 2 \in \partial \Sigma_{j}$, for $j=1,2$. The inverses of these functions will again be denoted by $z_{j}(w)$. Similar notation will denote inverses of defined functions. We define

$$
\omega(w)=\frac{e^{w}-1}{e^{w}+1}, \quad \zeta(z)=\frac{e^{z}-1}{e^{z}+1}
$$

in the appropriate domains, so that $\Sigma_{1}$ and $\Sigma_{2}$ correspond to the interior $\Omega$ and 
exterior $\Omega^{*}$ of a closed Jordan curve $\Gamma$, while $S_{1}$ and $S_{2}$ correspond to $D$ and $D^{*}$. Then $f(\zeta)=\omega\left(w_{1}(z(\zeta))\right)$ is a conformal mapping of $D$ onto $\Omega$, and $f^{*}(\zeta)=$ $\omega\left(w_{2}(z(\zeta))\right)$ maps $D^{*}$ conformally onto $\Omega^{*}$.

We shall construct the curves $C_{1}$ and $C_{2}$ so that $\Gamma$ is not an asymptotically conformal curve, but so that $f$ and $f^{*}$ are Lipschitz continuous. We begin by constructing "building blocks".

Choose and fix $\alpha \in(0, \pi / 2)$. For each integer $k \geqq 3$, consider the following circles in the $\omega=s+i t$ plane:

$$
\begin{gathered}
T_{1}:(t-\pi)^{2}+(s-k)^{2}=\left(\frac{\pi}{2}+\alpha\right)^{2}, \\
T_{2}: t^{2}+\left(s-u_{2}\right)^{2}=\frac{\pi^{2}}{4}, \quad T_{3}: t^{2}+\left(s-u_{3}\right)^{2}=\frac{\pi^{2}}{4},
\end{gathered}
$$

where $u_{2}=k-\left(\alpha^{2}+2 \alpha \pi\right)^{1 / 2}$ and $u_{3}=k+\left(\alpha^{2}+2 \alpha \pi\right)^{1 / 2}$, so that $T_{1}$ is tangent to $T_{2}$ and $T_{3}$. Let $L=\{s+i t: s \geqq 0, t=\pi / 2\}$. We trace a curve $\Gamma_{k}$ as follows. Starting at $\pi i / 2$, move to the right, first on $L$ to $T_{2}$, then on $T_{2}$ to $T_{1}, T_{1}$ to $T_{3}, T_{3}$ to $L$ and then on $L$ to $+\infty$. Let $\Gamma_{k}^{\prime}$ be the reflection of $\Gamma_{k}$ across the $s$ axis and take $\Omega_{k}$ to be the domain bounded by $\Gamma_{k} \cup \Gamma_{k}^{\prime} \cup\{t i:|t| \leqq \pi / 2\}$.

Now let $w(\omega)=-i e^{-\omega}+w_{k}$ for $w_{k}=2 k+\pi i / 2$, and let $D_{k}$ be the image of $\Omega_{k}$ under this mapping. Let $\Sigma=\{w=u+i v:|v|<\pi / 2\}$. Delete from $\Sigma$ the half disks $\left\{w:\left|w-w_{k}\right|<1, v<\pi / 2\right\}$ and replace them with the $D_{k}$. We christen the resulting domain $\Sigma_{1}$ and we let $\Sigma_{2}=\{w=u+i v:-\pi / 2<v<3 \pi / 2\}-\bar{\Sigma}_{1}$. The upper boundary of $\Sigma_{1}$ is then a curve $C_{1}$, with a sequence of double bumps going out to $+\infty$, decreasing in size. Under the mapping $\omega=\left(e^{w}+1\right) /\left(e^{w}-1\right), \Sigma_{1}$ is mapped onto a domain $\Omega$ which is nearly a unit disk, with a sequence of double bumps converging to $\omega=1$.

In order to show that $\partial \Omega$ is not asymptotically conformal, it suffices to consider the image of $\partial \Sigma_{1}$ under $W(w)=e^{-w}$, since

$$
\omega=\frac{1+e^{-w}}{1-e^{-w}}=\frac{1+W}{1-W}=h(W)
$$

and $h(W)$ is conformal at $W=0$.

For each $k(\geqq 3)$, let $w_{k}^{\prime}=-i e^{-k-i(\pi / 2-\alpha)}+w_{k}$ and $w_{k}^{\prime \prime}=-i e^{-k+i(\pi / 2-\alpha)}+w_{k}$. Consider the ratio

$$
\begin{gathered}
\frac{\left|W\left(w_{k}^{\prime}\right)-W\left(w_{k}\right)\right|+\left|W\left(w_{k}\right)-W\left(w_{k}^{\prime \prime}\right)\right|}{\left|W\left(w_{k}^{\prime}\right)-W\left(w_{k}^{\prime \prime}\right)\right|} \\
=\frac{\left|e^{-w_{k}^{\prime}}-e^{-w_{k}}\right|+\mid e^{-w_{k}}-e^{-w_{k}^{\prime \prime} \mid}}{\left|e^{-w_{k}^{\prime}}-e^{-w_{k}^{\prime \prime}}\right|}=\frac{\left|e^{w_{k}-w_{k}^{\prime}}-1\right|+\left|e^{w_{k}-w_{k}^{\prime \prime}}-1\right|}{\left|e^{w_{k}-w_{k}^{\prime}}-e^{w_{k}-w_{k}^{\prime \prime}}\right|} .
\end{gathered}
$$

Then $w_{k}-w_{k}^{\prime}=e^{-k}(\sin (\pi / 2-\alpha)+i \cos (\pi / 2-\alpha))=u+i v$ and $w_{k}-w_{k}^{\prime \prime}=-u+i v$. 
Furthermore $v=c u$ for $c=1 / \tan (\pi / 2-\alpha)$. Thus the quantity in (3.1) is

$$
\frac{\left|e^{-u+i c u}-1\right|+\left|e^{u+i c u}-1\right|}{\left(e^{u}-e^{-u}\right)}=\left(1+c^{2}\right)^{1 / 2}+o(1)
$$

as $k \rightarrow \infty$, so that $u \rightarrow 0$. Thus we see that

$$
\lim _{\left|\omega^{\prime}-\omega^{\prime \prime}\right| \rightarrow 0} \sup _{\omega \in \Gamma\left(\omega^{\prime}, \omega^{\prime \prime}\right)} \frac{\left|\omega^{\prime}-\omega\right|+\left|\omega-\omega^{\prime \prime}\right|}{\left|\omega^{\prime}-\omega^{\prime \prime}\right|} \geqq\left(1+c^{2}\right)^{1 / 2}=\frac{1}{\sin (\pi / 2-\alpha)},
$$

which may be made as large as desired by taking $\alpha$ near $\pi / 2$.

We must now show that $f$ and $f^{*}$ are uniformly Lipschitz continuous on $\partial D$. We shall consider only $f$, as the proof for $f^{*}$ is essentially the same. The Lipschitz continuity of $f$ follows from the following result.

Lemma 5. With the notations above, suppose that there exist positive constants $M_{1}, K_{1}$ and $K_{2}$ such that for almost every $z \in \partial S_{1}$ with $\operatorname{Re} z=x>M_{1}$ we have

$$
\left|\frac{d w_{1}}{d z}(z)\right|<K_{1}
$$

and for $u_{1}(z)=\operatorname{Re} w_{1}(z)$ we have

$$
x-u_{1}(z)<K_{2} .
$$

Then there exist positive $K_{3}$ and $\delta$ such that for almost every $\zeta \in \partial D$ with $|\zeta-1|<\delta$,

$$
\left|\frac{d f}{d \zeta}(\zeta)\right|<K_{3} \text {. }
$$

Proof. We have, for $\zeta \neq \pm 1, z=z(\zeta)$ and $w=w_{1}(z)$,

$$
\begin{gathered}
\left|\frac{d f}{d \zeta}\right|=\left|\frac{d \omega}{d w}\right|\left|\frac{d w_{1}}{d z}\right|\left|\frac{d z}{d \zeta}\right|=\left|\frac{2 e^{w}}{\left(e^{w}+1\right)^{2}}\right|\left|\frac{d w_{1}}{d z}\right|\left|\frac{\left(e^{z}+1\right)^{2}}{2 e^{z}}\right| \\
=\left|\frac{d w_{1}}{d z}\right|\left|e^{z-w}\right|\left|\frac{1+e^{-z}}{1+e^{-w}}\right|^{2}=\left|\frac{d w_{1}}{d z}\right| e^{x-u_{1}(z)}(1+o(1)) \text { as } x \rightarrow \infty .
\end{gathered}
$$

We first show that (3.3) holds. Recall the Ahlfors upper inequality proved in [5, Theorem 3]: For $u>0$ and $x_{1}(w)=\operatorname{Re} z_{1}(w)$;

$$
\bar{x}_{1}(u)-\underline{x}_{1}(0) \leqq \pi \int_{0}^{u} \frac{d t}{\theta_{1}(t)}+M_{2}
$$

where the constant $M_{2}$ depends only on $\alpha$ in our case. From this we see that

$$
x_{1}(w)-u \leqq \int_{0}^{u} \frac{\pi-\theta_{1}(t)}{\theta_{1}(t)} d t+M_{3}
$$

for $M_{3}=M_{2}+\underline{x}_{1}(0)$. Since $\theta(t)=\pi$ off of the intervals

$$
\left[2 k-e^{-k+\left(\alpha^{2}+2 \alpha \pi\right)^{1 / 2}}, 2 k+e^{-k+\left(\alpha^{2}+2 \alpha \pi\right)^{1 / 2}}\right]
$$


and

$$
0 \leqq \pi-\theta_{1}(t) \leqq e^{-k+\left(\alpha^{2}+2 \alpha \pi\right)^{1 / 2}}
$$

on the corresponding interval, the integral in (3.5) converges as $u \rightarrow \infty$, and (3.3) follows.

We next note that the domain $\Sigma_{1}$ is such that the mapping $z_{1}(w)=x_{1}(w)+i y_{1}(w)$ from $\Sigma_{1}$ onto $S_{1}$ is "semiconformal" [14]:

For any $r, 0<r<\pi / 2$, there exists $u_{r}$ such that

$$
S_{1} \supset S_{r}=\left\{u+i v: u \geqq u_{r},-\frac{\pi}{2}+r<v<\frac{\pi}{2}-r\right\}
$$

and

$$
\lim _{\substack{w \rightarrow+\infty \\ w \in S_{r}}} y_{1}(w)-v=0
$$

This will be used in showing that (3.2) holds for $w_{1}$. The proof of (3.2) is somewhat delicate, and we must consider properties of the $\Omega_{k}$.

For fixed $k \geqq 3$, let $z_{k}=z_{1}\left(w_{k}\right)$ and define $\zeta(z)=\log \left(1 /\left(z-z_{k}\right)\right)-(\pi / 2) i$ for $z \in S_{1}$, so that $|\operatorname{Im} \zeta(z)<\pi / 2|$. Let $\omega(w)=\log \left(1 /\left(w-w_{k}\right)\right)-(\pi / 2) i$ for $w \in D_{k}$. Then $\zeta_{k}(\omega)=\zeta\left(z_{1}(w(\omega))\right)$ maps $\Omega_{k}$ conformally onto a strip domain $S_{k}$ which is bounded by the lines

and

$$
\left\{\zeta=\xi+i \frac{\pi}{2}: \xi \geqq \log \left|\frac{1}{z_{1}\left(w_{k}-1\right)-z_{k}}\right|\right\}
$$

$$
\left\{\zeta=\xi-i \frac{\pi}{2}: \xi \geqq \log \left|\frac{1}{z_{1}\left(w_{k}+1\right)-z_{k}}\right|\right\}
$$

and by a curve $\gamma_{k}$ connecting the left endpoints of the lines. Observe that for $\zeta \in \gamma_{k},|\operatorname{Im} \zeta| \leqq \pi / 2$.

Lemma 6. Let $\zeta_{k}(\omega)=\xi_{k}(\omega)+i \eta_{k}(\omega)$ map $\Omega_{k}$ onto $S_{k}$ as above. Then there exists $M_{1}$ such that for all $k \geqq 3$,

$$
\underline{\xi}_{k}(0) \leqq M_{1}
$$

Furthermore, there exists $M_{2}$ depending only on $\alpha$ such that for $\omega=s>M_{2}$

$$
\left|\eta_{k}(s)\right|<(\pi / 2+\alpha) / 2<\pi / 2, \text { for all } k \text {. }
$$

Proof. Consider $\zeta_{k}^{\prime}=\zeta\left(z_{1}\left(w_{k}-i\right)\right) \in \gamma_{k}$. By (3.6), $\operatorname{Im}\left(z_{1}\left(w_{k}-i\right)\right) \rightarrow \pi / 2-1$ as $k \rightarrow \infty$, so that $\left|z_{1}\left(w_{k}-i\right)-z_{k}\right|>1 / 2$ for $k$ sufficiently large. From this it follows that $\xi_{k}(0) \leqq \operatorname{Re} \zeta_{k}^{\prime}<\log 2$ for large $k$ and (3.7) then follows.

$\bar{N}$ ext, let $h_{k}(\omega)=\eta_{k}(\omega)-t$. Then $h_{k}$ is harmonic in $\Omega_{k}$ and for $\omega=i t \in \partial \Omega_{k}$, $|t|<\pi / 2$, we have $\left|h_{k}(\omega)\right|<\pi$. On the rest of $\partial \Omega_{k},\left|h_{k}(\omega)\right| \leqq \alpha$. Thus, for any $\varepsilon>0$, the two constants theorem and a standard comparison argument show that there exists $K>0$ for which $\operatorname{Re} \omega>K$ implies that $\left|h_{k}(\omega)\right| \leqq \alpha+\varepsilon$. We let $\omega=s$ and $\varepsilon=(\pi / 2-\alpha) / 2$ to obtain (3.8). 
Next we state a result which will be applied to the mapping $w_{1}(z)$ and to the $\omega_{k}(\zeta)$. We state it for $w_{1}$. The proof is essentially that of Lemma 3 in [6].

Lemma 7. Let $w_{1}(z)$ map $S_{1}$ conformally onto $\Sigma_{1}$ as above. Let $w=w_{1}(z) \epsilon$ $\partial \Sigma_{1}$ and let $r>0$ and $M>0$ be such that:

(3.9) There exist circles $T$ and $T^{\prime}$ with radius $r$ and tangent to $\partial \Sigma_{1}$ at $w$, with $T \subset \bar{\Sigma}_{1}$ and $T^{\prime} \subset \overline{\left(-\Sigma_{1}\right)}$.

(3.10) The center of $T$ is $w_{0}=w_{1}\left(z_{0}\right)$, and $\left|\operatorname{Im} z_{0}\right| \leqq M<\pi / 2$.

Then there exists $K>0$, depending only on $r$ and $M$, such that

$$
\left|\frac{d w_{1}}{d z}(z)\right|<K \text {. }
$$

Lemmas 6 and 7 will be used to prove

Lemma 8. There exist positive constants $\delta$ and $K^{\prime}$, depending only on $\alpha$, such that for $w \in C_{1}$ with $0<\left|w-w_{k}\right|<\delta$ for any $k \geqq 3$, we have, for $z=z_{1}(w)$

$$
\left|\frac{d w_{1}}{d z}(z)\right|<K^{\prime} \text {. }
$$

Proof. We shall establish that there exist constants $M, K_{1}$ and $K_{2}$ such that for $\zeta \in \partial S_{k}$ with $\xi=\operatorname{Re} \zeta>M$, we have

we have

$$
\left|\frac{d \omega_{k}}{d \zeta}(\zeta)\right|<K_{1}, \quad \text { and for } \quad s_{k}(\zeta)=\operatorname{Re} \omega_{k}(\zeta)
$$

$$
\xi-s_{k}(\xi)<K_{2}
$$

We begin by applying Ahlfors' upper inequality to the mapping $\zeta_{k}(\omega)$ to see that there exists $M_{2}$, depending only on $\alpha$ such that

By (3.7) and the construction of $\Omega_{k}$,

$$
\bar{\xi}_{k}(s)-\underline{\xi}_{k}(0) \leqq \int_{0}^{s} \frac{\pi}{\theta(\tau)} d \tau+M_{2} .
$$

$$
\xi_{k}(\omega)-s \leqq M_{1}+M_{2}+\int_{0}^{s} \frac{\pi-\theta(\tau)}{\theta(\tau)} d \tau \leqq K_{2}
$$

so that (3.12) holds.

Next we may suppose that $\omega=\omega_{k}(\zeta) \in \Gamma$ with $\operatorname{Re} \omega>\pi / 2$. Then there exist circles $T$ and $T^{\prime}$ which are tangent to $\Gamma_{k}$ at $\omega$ and whose interiors are respectively interior and exterior to $\Omega_{k}$. We take the center of $T$ to be at $s_{0}$ on the $s$ axis, and the radius of both circles to be $r \geqq(\pi / 2)-\alpha$. By Lemma 6 there exists $M>0$ such that for $s_{0}>M,\left|\eta_{k}\left(s_{0}\right)\right| \leqq((\pi / 2)+\alpha) / 2<\pi / 2$. Then apply the version of Lemma 7 for the mappings $\omega_{k}(\zeta)$ between half strips to establish (3.11), where $K_{1}$ depends only on $r$ and $M$, which in turn depend only on $\alpha$. Lemma 8 is then proved by the argument in the proof of Lemma 5 . 
We may now show that (3.2) holds. Let $\delta<1$ be as in Lemma 8 and let $N(\geqq 3)$ be such that for $k \geqq N$, exp $\left(-k+\left(\alpha^{2}+2 \alpha \pi\right)^{1 / 2}\right)<\delta / 2$. Let $A=\left\{w \in C_{1}: 0<\left|w-w_{k}\right|<\delta\right.$, $k \geqq N\}$ and let $B=\left\{w \in \partial S_{1}: \operatorname{Re} w \geqq 2 N, w \in A \cup\left\{w_{k}\right\}\right\}$. Then any point of $B$ is on one of the lines $v=\pi / 2$ or $v=-\pi / 2$, and for any such point, the condition (3.9) holds with $r=\delta / 2$. Because of (3.6), there exists $M_{1}>2 N$ such that (3.10) holds for $w \in B$ with $\operatorname{Re} w \geqq M_{1}$. Thus (3.2) holds for almost every $w \in \partial S_{1}$ for which $\operatorname{Re} w \geqq M_{1}$ and with $K_{1}=\max \left(K, K^{\prime}\right)$. Thus Lemma 5 may be applied to $w_{1}(z)$ and it finally follows that $f$ is Lipschitz continuous on $\partial D$.

\title{
References
}

[1] Ahlfors, L. V.: Lectures on quasiconformal mappings. - D. Van Nostrand Company, Inc., Princeton, New Jersey-Toronto-New York-London, 1966.

[2] Becker, J., and Сн. Pommerenke: Über die quasiconforme Fortsetzung schlichter Funktionen. - Math. Z. 161, 1978, 69-80.

[3] BeCKer, J., and CH. Pommerenke: Hölder continuity of conformal mappings and non-quasiconformal Jordan curves. - Comment. Math. Helv., 57, 1982, 221-225.

[4] EKe, B. G.: Remarks on Ahlfors' distortion theorem. - J. Analyse Math. 19, 1967, 97-134.

[5] Jenkins, J. A., and K. OIKAWA: On results of Ahlfors and Hayman. - Illinois J. Math. 15, 1971, 664-671.

[6] LesLey, F. D.: On interior and exterior conformal mappings of the disk. - J. London Math. Soc. (2) $20,1979,67-78$.

[7] LESLEY, F. D.: Hölder continuity of conformal mappings at the boundary via the strip method. - Indiana Univ. Math. J. 31, 1982, 341-354.

[8] LeSLey, F. D., and S. E. WARSCHAwsKi: Boundary behavior of the Riemann mapping function of asymptotically conformal curves. - Math. Z. 179, 1982, 299-323.

[9] NÄKkI, R., and B. PALKA: Quasiconformal circles and Lipschitz classes. - Comment. Math. Helv. 55, 1980, 485-498.

[10] Pommerenke, Ch.: On univalent functions, Bloch functions and VMOA. - Math. Ann. 236, 1978, 199-208.

[11] Rodin, B., and S. E. WARSCHAwski: Extremal length and univalent functions III. Consequences of the Ahlfors distortion property. - Bull. Inst. Math. Acad. Sinica 6, 1978, 583-597.

[12] Rodin, B., and S. E. WARSCHAwsKi: On the derivative of the Riemann mapping function near a boundary point and the Visser-Ostrowski problem. - Math. Ann. 248, 1980, 125-137.

[13] WARSCHAWSKI, S. E.: On the degree of variation in conformal mapping of variable regions. Trans. Amer. Math. Soc. 69, 1950, 335-356.

[14] WaRschawski, S. E.: On the boundary behavior of conformal maps. - Nagoya Math. J. 30, $1967,83-101$.

\author{
San Diego State University \\ Department of Mathematics \\ San Diego, California 92182 \\ USA
}

Received 25 January 1983 